\title{
Pregnancy in a Patient with Congenital Complete Transposition of Great Arteries after Atrial Switch Operation: A Case Report with a Review of Literatures
}

\author{
Jung Un Shinn¹, Hyun Hwa Cha*1, Yeo Hyang Kim² and Won Joon Seong ${ }^{1}$ \\ ${ }^{1}$ Department of Obstetrics and Gynecology, Kyungpook National University Hospital, South Korea \\ ${ }^{2}$ Department of Pediatrics, Kyungpook National University, South Korea
}

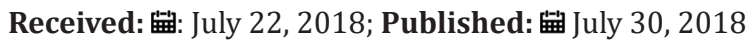

*Corresponding author: Hyun Hwa Cha, Department of Obstetrics and Gynecology, Kyungpook National University Hospital, School of Medicine, Kyungpook National University 807 Hoguk-ro, Buk-gu 702-720, Daegu, South Korea.

\begin{abstract}
Complete transposition of the great arteries (d-TGA) accounting for $5 \%$ of all congenital heart disease (CHD) is one of the most common cyanotic CHDs. Advances in congenital cardiology and surgery improve the prognosis in patients with TGA, nowadays women who survived the disease are entering the child bearing ages. Although, arterial switch operation has been becoming the standard treatment for TGA, atrial switch operation (ASO) was performed till past decades. Right ventricle (RV) becomes to be in charge of systemic circulation in patients with ASO. RV cannot sustain the demands of the systemic circulation and gradually decrease the function. Recently, we have experienced a pregnancy in a patient with congenital TGA treated with ASO. She uneventfully delivered a male neonate weighing of 3100 grams at 38 weeks of gestation. However, she developed the sign of heart failure on four days after delivery. We successfully managed her with administration of oxygen, diuretics and angiotensin converting enzyme (ACE) inhibitor. Herein, we report this case with a review of literatures.

Abbreviations: CHD: Congenital Heart Diseases; ASO: Atrial Switch Operation; RV: Right Ventricle; ACE: Angiotensin Converting Enzyme; SVR: Systemic Vascular Resistance; TR: Tricuspid Regurgitation; NYHA: Heart Association; MRI: Magnetic Resonance Imaging; USG: Obstetrical Ultrasonography; FS: Fractional Shortening; ICU: Intensive care unit; CSE: Combined Spinal and Epidural; PACU: Post Anesthesia Care Unit; SVC: Superior Vena Cava; ICV: Inferior Vena Cava
\end{abstract}

\section{Introduction}

Congenital heart diseases (CHD) is one of the most common congenital anomalies with an incidence of about 8/1000 live births [1]. Complete transposition of great arteries (d-TGA) is a relatively frequent cardiac anomaly occurring in $5 \%$ to 7 of all congenital cardiac malformations [2]. Since its spontaneous mortality within the first 2 years is $90 \%$ without surgical intervention, almost all pregnant women with TGA have undergone one form of the surgical repairs such as atrial switch operation (ASO), arterial switch operation or extra conduit repair (Rastelli) [3]. Although ASO can restore a physiologic circulation, the morphological RV should sustain the systemic circulation [3,4]. Physiologic hemodynamic changes during pregnancy, delivery and immediate postpartum period could result in deteriorating of systemic RV functions and/ or tricuspid valve regurgitation [4]. Herein, we aimed to report a case of pregnancy with congenital TGA treated with ASO.

\section{Case Presentations}

A 28-year-old woman (gravida 1, para 0) visited our institution for antenatal care. She was diagnosed as complete TGA postnatally and had undergone balloon atrial septostomy after birth, and subsequently underwent Senning's operation at the age of 1-yearold. She has been administered angiotensin converting enzyme (ACE) inhibitor (Enalapril 5mg/day) for improving diastolic function and decreasing systemic vascular resistance (SVR). Her regular 2D echocardiography showed normal Tei index for both ventricles (Left ventricle: 1\%, Right ventricle: $32 \%$ ) and grade I Tricuspid Regurgitation (TR) without arrhythmia. Also, her New York Heart Association (NYHA) functional classification was class I. She was planned to perform cardiac magnetic resonance imaging (MRI) for counseling the pregnancy risk ahead of marriage; however, she got pregnant unexpectantly.

At her first time of visiting obstetrical unit, obstetrical ultrasonography (USG) revealed a fetus measuring of 83/7 weeks of gestation. We recommended the cessation of ACE inhibitor administration and decided to delay cardiac MRI after delivery. We performed 2D echocardiography at 13 weeks of gestation showing $35 \%$ of RV fractional shortening (FS) in RV with patent Senning pathway (Figure 1). We regularly checked the level of $\mathrm{N}$-terminal probrain natriuretic peptide (NT-proBNP) for detection 
of heart failure (Figure 2) and performed carefully obstetrical care. 2D echocardiography performed at $373 / 7$ weeks revealed that decreased RV function compared to previous study (FS 22\%). There was no significant event during antepartum period. We discussed about the mode of delivery with the pediatric cardiologist and obstetrical anesthesiologist. We thought that elective delivery would be better; therefore, we decided to perform cesarean delivery at $381 / 7$ weeks of gestation.

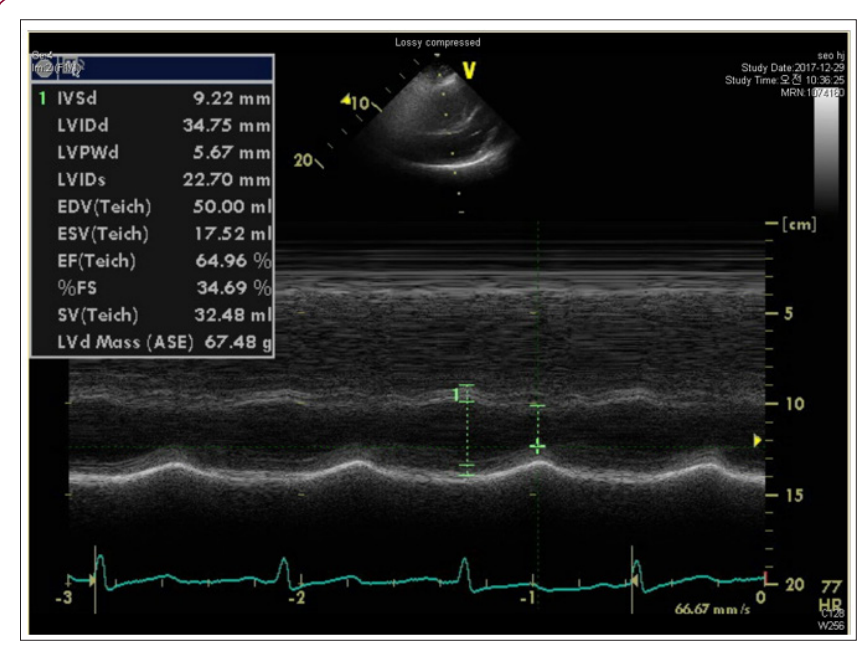

Figure 1: Maternal 2D echocardiography showed that functional fraction of the maternal right ventricle was $35 \%$.

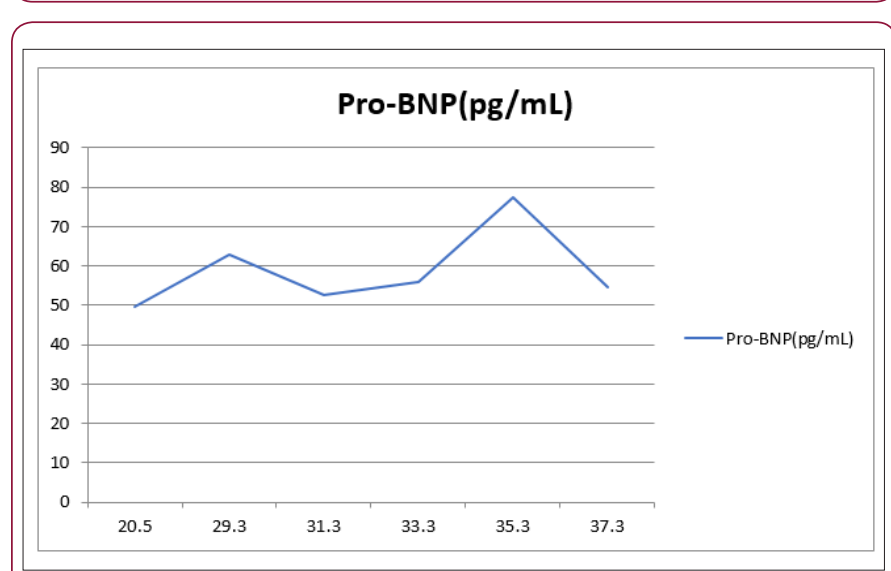

Figure 2: he levels of maternal NT-Pro-BNP during antepartum period. They remained stable during the pregnancy.

A male neonate was born weighing 3100 gram with Apgar score 8 and 9 in 1-minute and 5-minutes. The anesthesiologist chose combined spinal and epidural (CSE) method to avoid a sudden change in blood pressure. An arterial cannulation was established at her right radial artery for close monitoring of blood pressure and continuous cardiac output. Her blood pressure maintained the range of 110-120/69-85 mmHg during systolic and diastolic period, respectively. Also, continuous electrocardiogram showed normal sinus rhythm without arrhythmia at operative room and post anesthesia care unit (PACU). However, her postoperative NTproBNP levels started to increase abruptly on 3rd day after delivery (Figure 3). The level of NT-proBNP more increased on 4th day after delivery and she began to complain chest tightness, dyspnea and sweating. We decided to admit her in the intensive care unit (ICU) and started applying of oxygen, administering ACE inhibitor (5 $\mathrm{mg}$ /day) and diuretics, of which start dose was furosemide $20 \mathrm{mg} /$ day intravenously followed by $10 \mathrm{mg}$ twice/day and $5 \mathrm{mg}$ /day. Her symptom was improved and NT-proBNP level was decreased to normal value. She was discharged with good condition on 8th day after delivery and will undergo cardiac MRI near soon.

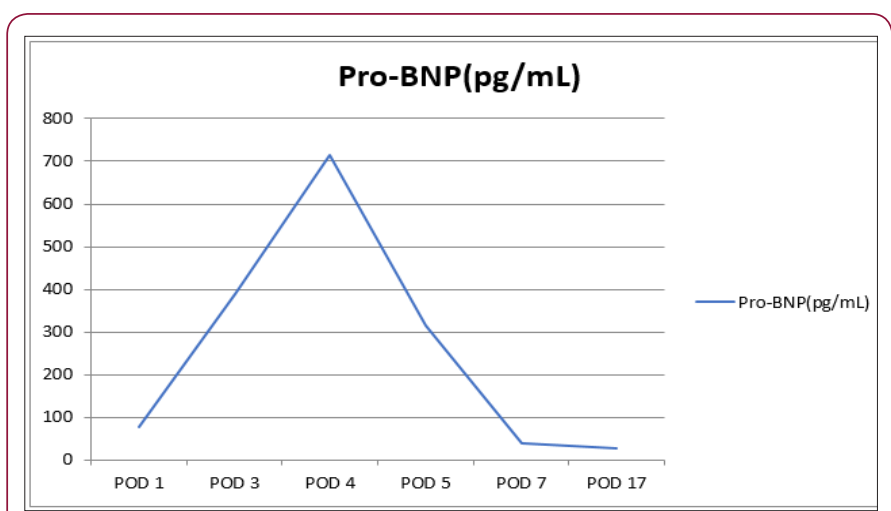

Figure 3: The levels of maternal NT-Pro-BNP during postpartum period. Her postoperative NT-proBNP levels started to increase abruptly on $3^{\text {rd }}$ day after delivery.

\section{Discussion}

Atrial switch operation (ASO) includes the "Senning operation" introduced in 1957 and the "Mustard operation" introduced in 1964 [5]. Senning operation created a conduit that routes the deoxygenated blood from the superior vena cava (SVC) and inferior vena cava (ICV) into the mitral valve and left ventricle [5]. By contrast, Mustard operation excised the atrial septum and created a baffle out of prosthetic material in 1963 [5]. Since these methods force the morphological RV to act as systemic pump, pregnant women who performed ASO would be at high risk during their pregnancies [6]. Pregnancy is usually related with prominent hemodynamic changes including increase of stroke volume, plasma volume, heart rate and cardiac output [6]. Although, studies about the obstetrical and cardiac outcomes in women with ASO are limited due to the rarity of this situation, previous studies reported that ASO would be related with obstetrical complications including preterm birth, intrauterine growth restriction and cardiac complication such as deterioration of RV function, arrhythmia, or deterioration of NYHA functional classification $[7,8]$.

One of the most common cardiac complications in women with ASO is arrhythmia such as sinus node dysfunction, atrial and ventricular tachyarrhythmia, which occurs with $14 \%-20 \%$ of incidence $[6,8]$. In addition to atrial scar formed during atrial repair, increased circulatory burden during pregnancy would be one of the trigger factors [5,8]. Therefore, arrhythmia should be concerned in pregnant patients with ASO. Though other cardiac complications are known to be uncommon, RV failure with progressive tricuspid regurgitation was developed in $10-25 \%$ of pregnant women with ASO $[4,7]$. The ventricular pressure over time $(\mathrm{dP} / \mathrm{dt})$ is an indirect measure of the force of the ventricle that requires the presence of some degree of TR to be assessed contraction. It is more reliable methods for qualifying systemic RV function [7] but we assessed the RV function by Tei and FS due to her poor echo window. Meanwhile, 
baffle obstruction is also important cardiac complication. Overall the rate of baffle obstruction was known to be $5 \%$ after ASO [9]; however, the rate of baffle obstruction during pregnancy in patent with ASO was 36\% [7].

Therefore, the authors suggested that even if a patient has no symptom, screening for baffle obstruction is necessary before a pregnancy. And baffle stenting should be considered before pregnancy for any degree of baffle narrowing, because obstruction is likely to worsen during pregnancy [9]. It is not clear which factors determine the prognosis of pregnant women with ASO. We suggested that early age, stable NYHA functional class (I or II) and normal levels of NT-proBNP would be good prognosis factors. A previous report also showed that patients whose NT-proBNP level was higher than $200 \mathrm{pg} / \mathrm{mL}$ were more likely to have higher right ventricular diameter, or more likely to be under diuretic treatment [10]. In conclusion, we thought that a pregnancy could be manageable in some kind of patients with ASO. The careful obstetrical and cardiologic management are needed.

\section{References}

1. Abu Harb M, Hey E, Wren C (1994) Death in infancy from unrecognised congenital heart disease. Arch Dis Child 71(1): 3-7.

2. Abuhamad A, Chaoui R (2015) Diagnosis and management of adult

\section{ISSN: 2574-1241}

DOI: 10.26717/BJSTR.2018.07.001491

Hyun Hwa Cha. Biomed J Sci \& Tech Res

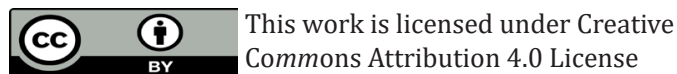

Submission Link: https://biomedres.us/submit-manuscript.php congenital heart disesase Curchill Livingstone pp. 447.

3. Trigas V, Nagglyman N, Pildner von Steinburg S, Oechslin E, Vogt M, et al. (2014) Pregnancy-related obstetric and cardiologic problems in women after atrial switch operation for transposition of the great arteries. Circ J 78(2): 439-443

4. Niwa K [2018] Adult Congenital Heart Disease with Pregnancy. Korean Circ J 48(4): 251-276.

5. Haeffele C, Lui GK (2015) Dextro-Transposition of the Great Arteries: Long-term Sequelae of Atrial and Arterial Switch. Cardiol Clin 33(4): 543-558.

6. Cataldo S, Doohan M, Rice K, Trinder J, Staurt AG, et al. [2016] Pregnancy following Mustard or Senning correction of transposition of the great arteries: a retrospective study. BJOG 123(5): 807-813.

7. Metz TD, Jackson GM, Yetman AT [2011] Pregnancy outcomes in women who have undergone an atrial switch repair for congenital d-transposition of the great arteries. Am J Obstet Gynecol 205(3): 273. e1-5.

8. Drenthen W, Pieper PG, Ploeg M, Voors AA, Roos-Hesselink JW, et al. [2005] Risk of complications during pregnancy after Senning or Mustard (atrial) repair of complete transposition of the great arteries. Eur Heart J 26(23): 2588-2595.

9. Wells WJ, Blackstone E [2000] Intermediate outcome after Mustard and Senning procedures: A study by the Congenital Heart Surgeons Society. Semin Thorac Cardiovasc Surg Pediatr Card Surg Annu 3: 186-197.

10. Martinez Quintana E, Marrero Negrin N, Gopar Gopar S, Rodriguez Gonzalez F [2017] Right ventricular function and N-terminal pro-

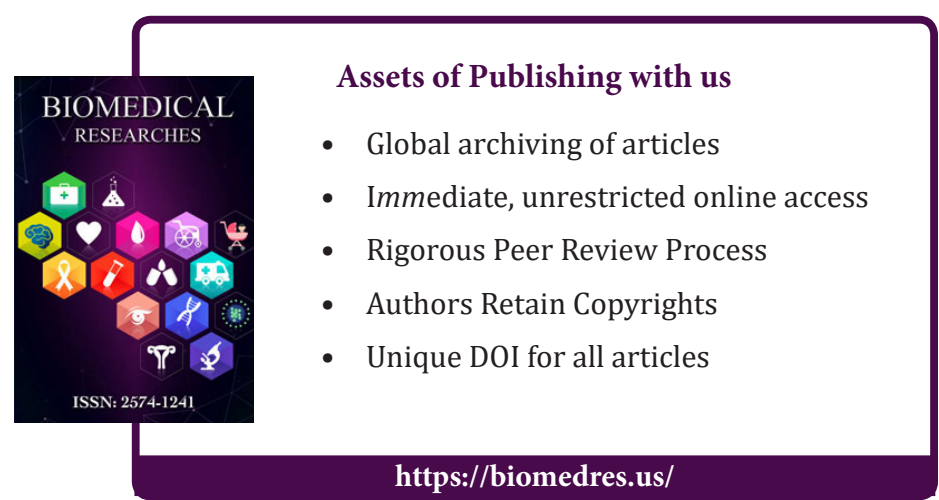

